CUBO A Mathematical Journal

Vol.12, Noㅡ, (19-27). June 2010

\title{
Differences of weighted composition operators between weighted Banach spaces of holomorphic functions and weighted Bloch type spaces
}

\author{
ELKE WOLF \\ Mathematical Institute, \\ University of Paderborn, \\ D-33095 Paderborn, Germany. \\ email: lichte@math.uni-paderborn.de
}

\begin{abstract}
We consider analytic self-maps $\phi_{1}, \phi_{2}$ of the open unit disk as well as analytic maps $\psi_{1}, \psi_{2}$. These maps induce differences of weighted composition operators acting between weighted Banach spaces of holomorphic functions and weighted Bloch type spaces. In this article we give necessary and sufficient conditions for such a difference to be bounded resp. compact.
\end{abstract}

\section{RESUMEN}

Nosotros consideramos auto aplicaciones $\phi_{1}, \phi_{2}$ del disco unitario abierto bien como aplicaciones analíticas $\psi_{1}, \psi_{2}$. Estas aplicaciones inducen diferencias de composición de operadores con peso actuando entre espacios de Banach pesados de funciones holomorfas y espacios de tipo Bloch con peso. En este artículo damos condiciones necesarias y suficientes para que tal diferencia sea acotada, respectivamente, compacta.

Key words and phrases: weighted composition operators, weighted Bloch type spaces, weighted Banach spaces of holomorphic functions.

Math. Subj. Class. 2000: 47B33, 47B38. 


\section{Introduction}

For analytic self-maps $\phi_{1}, \phi_{2}$ of $\mathbb{D}$ and analytic maps $\psi_{1}, \psi_{2}$ the corresponding weighted composition operators $\psi_{i} C_{\phi_{i}}$ are defined by $\psi_{i} C_{\phi_{i}} f=\psi_{i} f \circ \phi_{i}, i=1,2$. Composition operators and weighted composition operators acting on various spaces of analytic functions have recently been of much interest, see for example [14], [8], [12], [2], [4], [13]. Differences of them have been studied e.g. in [3], [9], [16], [17], [18].

Let $v$ and $w$ be strictly positive, continuous and bounded functions (weights) on $\mathbb{D}$ and $H(\mathbb{D})$ be the set of all analytic functions on $\mathbb{D}$. In this article we are interested in differences $\psi_{1} C_{\phi_{1}}-\psi_{2} C_{\phi_{2}}$ acting between weighted Banach spaces of holomorphic functions

$$
H_{v}^{\infty}:=\left\{f \in H(\mathbb{D}) ;\|f\|_{v}:=\sup _{z \in \mathbb{D}} v(z)|f(z)|<\infty\right\}
$$

and the weighted Bloch type spaces $B_{w}$ of functions $f \in H(\mathbb{D})$ satisfying $\|f\|_{B_{w}}:=\sup _{z \in \mathbb{D}} v(z)\left|f^{\prime}(z)\right|<\infty$.

Our aim is to give necessary and sufficient conditions for a difference $\psi_{1} C_{\phi_{1}}-\psi_{2} C_{\phi_{2}}: H_{v}^{\infty} \rightarrow B_{w}$ to be bounded resp. compact in terms of the involved weights and the analytic maps $\phi_{1}, \phi_{2}, \psi_{1}, \psi_{2}$.

\section{Notation and auxiliary results}

An introduction to the concept of composition operators can be found in the monographs [5] and [15]. In this article we are especially interested in radial weights (i.e. weights with $v(z)=v(|z|)$ for every $z \in \mathbb{D}$ ) which satisfy additionally the Lusky condition $(L 1)$ (due to Lusky [10])

$$
\text { (L1) } \inf _{k \in \mathbb{N}} \frac{v\left(1-2^{-k-1}\right)}{v\left(1-2^{-k}\right)}>0 \text {. }
$$

When dealing with differences of weighted composition operators we need some geometric data. Recall that for any $z \in \mathbb{D}, \varphi_{z}$ is the Möbius transformation which interchanges the origin and $z$, namely $\varphi_{z}(w)=\frac{z-w}{1-\bar{z} w}, w \in \mathbb{D}$. The pseudohyperbolic distance $\rho(z, w)$ for $z, w \in \mathbb{D}$ is defined by $\rho(z, w)=\left|\varphi_{z}(w)\right|$. Moreover, we have $\varphi_{z}^{\prime}(w)=\frac{|z|^{2}-1}{(1-\bar{z} w)^{2}}$ for $z, w \in \mathbb{D}$. Let us recall some auxiliary results.

The next lemma is taken from [3], see also [6].

Lemma 1. (Bonet-Lindström-Wolf [3]) Let $v$ be a radial weight satisfying the Lusky condition (L1) and let $f \in H_{v}^{\infty}$. Then there exists a constant $C_{v}>0$ (depending on the weight $v$ ) such that

$$
|f(z)-f(p)| \leq C_{v}\|f\|_{v} \max \left\{\frac{1}{v(z)}, \frac{1}{v(p)}\right\} \rho(z, p)
$$

for all $z, p \in \mathbb{D}$.

Theorem 2. (Harutyunyan-Lusky, [7] Theorem 2.1) Let $v$ and $w$ be radial weights which are continuously differentiable with respect to $|z|$ with $\lim _{|z| \rightarrow 1} v(z)=\lim _{|z| \rightarrow 1} w(z)=0$ and such that $H_{w}^{\infty}$ is isomorphic to $l_{\infty}$. If $\lim \sup _{r \rightarrow 1}\left(-\frac{w^{\prime}(r)}{v(r)}\right)<\infty$, then $D: H_{v}^{\infty} \rightarrow H_{w}^{\infty}, f \rightarrow f^{\prime}$ is bounded. 
For conditions when $H_{w}^{\infty}$ is isomorphic to $l_{\infty}$ we refer the reader to [11] and [7]. By [7] we know that the following weights have the desired properties:

$w(z)=(1-|z|)^{\alpha}, \alpha>0, w(z)=e^{-\frac{1}{1-|z|}}, z \in \mathbb{D}$.

For the study of the compactness of the difference $\psi_{1} C_{\phi_{1}}-\psi_{2} C_{\phi_{2}}$ we need the following result.

Proposition 3. (Cowen-MacCluer, [5] Proposition 3.11) Let $X$ and $Y$ be $H_{v}^{\infty}$ or $B_{w}$. Then $\psi_{1} C_{\phi_{1}}-$ $\psi_{2} C_{\phi_{2}}: X \rightarrow Y$ is compact if and only if for every bounded sequence $\left(f_{n}\right)_{n \in \mathbb{N}}$ in $X$ such that $f_{n} \rightarrow 0$ uniformly on the compact subsets of $\mathbb{D}$, then $\left(\psi_{1} C_{\phi_{1}}-\psi_{2} C_{\phi_{2}}\right) f_{n} \rightarrow 0$ in $Y$.

\section{Main Result}

In the sequel we consider weights $v$ of the following type: Let $\nu$ be a holomorphic function on $\mathbb{D}$, non-vanishing and strictly positive on $[0,1[$. Moreover we assume that $\nu$ is decreasing on $[0,1[$ and satisfies $\lim _{r \rightarrow 1} \nu(r)=0$. Then we define the corresponding weight $v$ by $v(z):=\nu\left(|z|^{2}\right)$ for every $z \in \mathbb{D}$. Furthermore, we suppose the boundedness of the function $\nu^{\prime}$ on $\mathbb{D}$. Next, we give some illustrating examples of weights of this type:

(i) Consider $\nu(z)=(1-z)^{\alpha}, \alpha \geq 1$. Then the corresponding weight is the so-called standard weight $v(z)=\left(1-|z|^{2}\right)^{\alpha}$.

(ii) Selecting $\nu(z)=e^{-\frac{1}{(1-z)^{\alpha}}}, \alpha \geq 1$, we obtain the weight $v(z)=e^{-\frac{1}{\left(1-|z|^{2}\right)^{\alpha}}}$.

(iii) Choose $\nu(z)=\sin (1-z)$ and the corresponding weight is given by $v(z)=\sin \left(1-|z|^{2}\right)$.

Fix a point $p \in \mathbb{D}$ and an analytic self-map $\phi$ of $\mathbb{D}$. We introduce a function

$$
v_{\phi(p)}(z):=\nu(\overline{\phi(p)} z) \text { for every } z \in \mathbb{D} \text {. }
$$

Since $\nu$ is holomorphic on $\mathbb{D}$, the function $v_{\phi(p)}$ is also holomorphic on $\mathbb{D}$. Furthermore, $v_{\phi(p)}(\phi(p))=$ $\nu\left(|\phi(p)|^{2}\right)=v(\phi(p))$ and $v_{\phi(p)}^{\prime}(z)=\overline{\phi(p)} \nu^{\prime}(\overline{\phi(p)} z)$ for every $z \in \mathbb{D}$, i.e. $v_{\phi(p)}^{\prime}(\phi(p))=\overline{\phi(p)} \nu^{\prime}\left(|\phi(p)|^{2}\right)$.

We start with considering boundedness of operators $\psi_{1} C_{\phi_{1}}-\psi_{2} C_{\phi_{2}}: H_{v}^{\infty} \rightarrow B_{w}$ and give first a necessary condition in terms of the involved weights and then a sufficient condition.

Proposition 4. Let $w$ be a weight and $v$ be a weight as described in the beginning of this section. Let $\psi_{1}, \psi_{2} \in H(\mathbb{D})$ and $\phi_{1}, \phi_{2}$ be analytic self-maps of $\mathbb{D}$. If $\psi_{1} C_{\phi_{1}}-\psi_{2} C_{\phi_{2}}: H_{v}^{\infty} \rightarrow B_{w}$ is bounded, then the following conditions are satisfied

(a) $\sup _{z \in \mathbb{D}} w(z)\left|\frac{\psi_{1}^{\prime}(z)}{v\left(\phi_{1}(z)\right)^{\frac{1}{2}}} \varphi_{\phi_{2}(z)}^{2}\left(\phi_{1}(z)\right)+2 \frac{\psi_{1}(z)}{v\left(\phi_{1}(z)\right)^{\frac{1}{2}}} \varphi_{\phi_{2}(z)}\left(\phi_{1}(z)\right) \varphi_{\phi_{2}(z)}^{\prime}\left(\phi_{1}(z)\right)\right|<\infty$,

(b) $\sup _{z \in \mathbb{D}} w(z)\left|\frac{\psi_{2}^{\prime}(z)}{v\left(\phi_{2}(z)\right)^{\frac{1}{2}}} \varphi_{\phi_{1}(z)}^{2}\left(\phi_{2}(z)\right)+2 \frac{\psi_{2}(z)}{v\left(\phi_{2}(z)\right)^{\frac{1}{2}}} \varphi_{\phi_{1}(z)}\left(\phi_{2}(z)\right) \varphi_{\phi_{1}(z)}^{\prime}\left(\phi_{2}(z)\right)\right|<\infty$,

(c) $\sup _{z \in \mathbb{D}}\left|\frac{\psi_{1}(z) w(z) \overline{\phi_{1}(z)} \nu^{\prime}\left(\left|\phi_{1}(z)\right|^{2}\right)}{v\left(\phi_{1}(z)\right)}\right| \rho\left(\phi_{1}(z), \phi_{2}(z)\right)<\infty$,

(d) $\sup _{z \in \mathbb{D}}\left|\frac{\psi_{2}(z) w(z) \overline{\phi_{2}(z)} \nu^{\prime}\left(\left|\phi_{2}(z)\right|^{2}\right)}{v\left(\phi_{2}(z)\right)}\right| \rho\left(\phi_{1}(z), \phi_{2}(z)\right)<\infty$, 
Proof (a) Fix a point $p \in \mathbb{D}$ and put

$$
f_{\phi_{1}(p)}(z):=\left(\frac{2}{v_{\phi_{1}(p)}(z)}-\frac{v_{\phi_{1}(p)}\left(\phi_{1}(p)\right)}{v_{\phi_{1}(p)}(z)^{2}}\right)^{\frac{1}{2}}
$$

and

$$
g_{\phi_{1}(p)}(z):=f_{\phi_{1}(p)}(z) \varphi_{\phi_{2}(p)}^{2}(z) \text { for every } z \in \mathbb{D}
$$

Next, we get

$$
\left\|g_{\phi_{1}(p)}\right\|_{v} \leq \sup _{z \in \mathbb{D}}\left|v(z)^{2} \frac{2}{v_{\phi_{1}(p)}(z)}-v(z)^{2} \frac{v_{\phi_{1}(p)}\left(\phi_{1}(p)\right)}{v_{\phi_{1}(p)}(z)^{2}}\right|^{\frac{1}{2}} \leq(3 M)^{\frac{1}{2}}
$$

where $M=\sup _{z \in \mathbb{D}} v(z)$ and therefore the constant does not depend on the choice of $p$. Thus, $g_{\phi_{1}(p)} \in$ $H_{v}^{\infty}$ and $g_{\phi_{1}(p)}^{\prime}(z)=f_{\phi_{1}(p)}^{\prime}(z) \varphi_{\phi_{2}(p)}^{2}(z)+2 f_{\phi_{1}(p)}(z) \varphi_{\phi_{2}(p)}^{\prime}(z) \varphi_{\phi_{2}(p)}(z)$ for every $z \in \mathbb{D}$, where $f_{\phi_{1}(p)}^{\prime}(z)=$ $\left(-\frac{v_{\phi_{1}(p)}^{\prime}(z)}{v_{\phi_{1}(p)}(z)^{2}}+\frac{v_{\phi_{1}(p)}^{\prime}(z) v_{\phi_{1}(p)}\left(\phi_{1}(p)\right)}{v_{\phi_{1}(p)}(z)^{3}}\right)\left(\frac{2}{v_{\phi_{1}(p)}(z)}-\frac{v_{\phi_{1}(p)}\left(\phi_{1}(p)\right)}{v_{\phi_{1}(p)}(z)^{2}}\right)^{-\frac{1}{2}}$ and $\varphi_{\phi_{2}(p)}^{\prime}(z)=\frac{\left|\phi_{2}(p)\right|^{2}-1}{\left(1-\overline{\phi_{2}(p)} z\right)^{2}}$ for every $z \in \mathbb{D}$. We get $f_{\phi_{1}(p)}\left(\phi_{1}(p)\right)=\frac{1}{v\left(\phi_{1}(p)\right)^{\frac{1}{2}}}$ and $f_{\phi_{1}(p)}^{\prime}\left(\phi_{1}(p)\right)=0$ and hence $g_{\phi_{1}(p)}\left(\phi_{1}(p)\right)=\frac{\varphi_{\phi_{2}(p)}^{2}\left(\phi_{1}(p)\right)}{v\left(\phi_{1}(p)\right)^{\frac{1}{2}}}$ as well as $g_{\phi_{1}(p)}^{\prime}\left(\phi_{1}(p)\right)=2 \frac{\varphi_{\phi_{2}(p)}^{\prime}\left(\phi_{1}(p)\right) \varphi_{\phi_{2}(p)}\left(\phi_{1}(p)\right)}{v\left(\phi_{1}(p)\right)^{\frac{1}{2}}}$. Now,

$$
\begin{array}{r}
w(p)\left|\frac{\psi_{1}^{\prime}(p) \varphi_{\phi_{2}(p)}^{2}\left(\phi_{1}(p)\right)}{\left.v\left(\phi_{1}(p)\right)\right)^{\frac{1}{2}}}+2 \frac{\psi_{1}(p) \varphi_{\phi_{2}(p)}\left(\phi_{1}(p)\right) \varphi_{\phi_{2}(p)}^{\prime}\left(\phi_{1}(p)\right)}{\left.v\left(\phi_{1}(p)\right)\right)^{\frac{1}{2}}}\right| \\
=w(p)\left|\psi_{1}^{\prime}(p) g_{\phi_{1}(p)}\left(\phi_{1}(p)\right)+\psi_{1}(p) \phi_{1}^{\prime}(p) g_{\phi_{1}(p)}^{\prime}\left(\phi_{1}(p)\right)-\psi_{2}^{\prime}(p) g_{\phi_{1}(p)}\left(\phi_{2}(p)\right)-\psi_{2}(p) \phi_{2}^{\prime}(p) g_{\phi_{1}(p)}^{\prime}\left(\phi_{2}(p)\right)\right| \\
\leq\left\|\psi_{1} C_{\phi_{1}}-\psi_{2} C_{\phi_{2}}\right\|\left\|g_{\phi_{1}(p)}\right\|_{v}<\infty .
\end{array}
$$

Thus, (a) follows, and we can show (b) analogously. For the proof of condition (c) we fix a point $p \in \mathbb{D}$ and put

$$
f_{\phi_{1}(p)}(z):=\frac{v_{\phi_{1}(p)}\left(\phi_{1}(p)\right)}{v_{\phi_{1}(p)}(z)}-\left(\frac{v_{\phi_{1}(p)}\left(\phi_{1}(p)\right)}{v_{\phi_{1}(p)}(z)}\right)^{\frac{1}{2}}=\frac{v\left(\phi_{1}(p)\right)}{v_{\phi_{1}(p)}(z)}-\left(\frac{v\left(\phi_{1}(p)\right)}{v_{\phi_{1}(p)}(z)}\right)^{\frac{1}{2}}
$$

and

$$
g_{\phi_{1}(p)}(z):=f_{\phi_{1}(p)}(z) \varphi_{\phi_{2}(p)}^{2}(z) \text { for every } z \in \mathbb{D} .
$$

Hence $\left\|g_{\phi_{1}(p)}\right\|_{v} \leq 2 M$ and we get

$$
g_{\phi_{1}(p)}^{\prime}(z)=f_{\phi_{1}(p)}^{\prime}(z) \varphi_{\phi_{2}(p)}^{2}(z)+2 f_{\phi_{1}(p)}(z) \varphi_{\phi_{2}(p)}(z) \varphi_{\phi_{2}(p)}^{\prime}(z) \text { for every } z \in \mathbb{D},
$$

where

$$
f_{\phi_{1}(p)}^{\prime}(z)=-\frac{v_{\phi_{1}(p)}\left(\phi_{1}(p)\right) v_{\phi_{1}(p)}^{\prime}(z)}{v_{\phi_{1}(p)}(z)^{2}}+\frac{1}{2} \frac{v_{\phi_{1}(p)}\left(\phi_{1}(p)\right)^{\frac{1}{2}} v_{\phi_{1}(p)}^{\prime}(z)}{v_{\phi_{1}(p)}(z)^{\frac{3}{2}}}
$$

Thus, we obtain $f_{\phi_{1}(p)}\left(\phi_{1}(p)\right)=0$ and $f_{\phi_{1}(p)}^{\prime}\left(\phi_{1}(p)\right)=-\frac{1}{2} \frac{\overline{\phi_{1}(p)} \nu^{\prime}\left(\left|\phi_{1}(p)\right|^{2}\right)}{v\left(\phi_{1}(p)\right)}$. Hence $g_{\phi_{1}(p)}\left(\phi_{1}(p)\right)=0$ and $g_{\phi_{1}(p)}^{\prime}\left(\phi_{1}(p)\right)=-\frac{1}{2} \frac{\overline{\phi_{1}(p)} \nu^{\prime}\left(\left|\phi_{1}(p)\right|^{2}\right) \varphi_{\phi_{2}(p)}^{2}\left(\phi_{1}(p)\right)}{v\left(\phi_{1}(p)\right)}$. Finally,

$$
\begin{array}{r}
\frac{1}{2} w(p)\left|\frac{\overline{\phi_{1}(p)} \nu^{\prime}\left(\left|\phi_{1}(p)\right|^{2}\right) \varphi_{\phi_{2}(p)}^{2}\left(\phi_{1}(p)\right)}{v_{\phi_{2}(p)}\left(\phi_{1}(p)\right)}\right| \\
=w(p)\left|\psi_{1}^{\prime}(p) g_{\phi_{1}(p)}\left(\phi_{1}(p)\right)+\psi_{1}(p) \phi_{1}^{\prime}(p) g_{\phi_{1}(p)}^{\prime}\left(\phi_{1}(p)\right)-\psi_{2}^{\prime}(p) g_{\phi_{1}(p)}\left(\phi_{2}(p)\right)-\psi_{2}(p) \phi_{2}^{\prime}(p) g_{\phi_{1}(p)}^{\prime}\left(\phi_{2}(p)\right)\right| \\
\leq\left\|\psi_{1} C_{\phi_{1}}-\psi_{2} C_{\phi_{2}}\right\|\left\|g_{\phi_{1}(p)}\right\|_{v}<\infty .
\end{array}
$$


The claim follows. We can show (d) analogously.

Proposition 5. Let $v$ and $w$ be weights. If

(a) there is a weight $u$ such that the operator $D: H_{v}^{\infty} \rightarrow H_{u}^{\infty}, f \rightarrow f^{\prime}$ is bounded and additionally $\sup _{z \in \mathbb{D}} \max \left\{\frac{w(z)}{u\left(\phi_{1}(z)\right)}, \frac{w(z)}{u\left(\phi_{2}(z)\right)}\right\} \rho\left(\phi_{1}(z), \phi_{2}(z)\right)<\infty$ as well as $\sup _{z \in \mathbb{D}} \max \left\{\frac{w(z)}{u\left(\phi_{1}(z)\right)}, \frac{w(z)}{u\left(\phi_{2}(z)\right)}\right\}\left|\phi_{1}^{\prime}(z) \psi_{1}(z)-\phi_{2}^{\prime}(z) \psi_{2}(z)\right|<\infty$,

(b) $\sup _{z \in \mathbb{D}} \max \left\{\frac{1}{v\left(\phi_{1}(z)\right)}, \frac{1}{v\left(\phi_{2}(z)\right)}\right\} w(z)\left|\psi_{1}^{\prime}(z)-\psi_{2}^{\prime}(z)\right|<\infty$,

(c) $\sup _{z \in \mathbb{D}} \max \left\{\frac{1}{v\left(\phi_{1}(z)\right)}, \frac{1}{v\left(\phi_{2}(z)\right)}\right\} w(z) \max \left\{\left|\psi_{1}^{\prime}(z)\right|,\left|\psi_{2}^{\prime}(z)\right|\right\} \rho\left(\phi_{1}(z), \phi_{2}(z)\right)<\infty$

then $\psi_{1} C_{\phi_{1}}-\psi_{2} C_{\phi_{2}}: H_{v}^{\infty} \rightarrow B_{w}$ is bounded.

Proof Let $f \in H_{v}^{\infty}$. Using Lemma 1 we obtain

$$
\begin{array}{r}
\sup _{z \in \mathbb{D}} w(z)\left|\left(\left(\psi_{1} C_{\phi_{1}}-\psi_{2} C_{\phi_{2}}\right) f\right)^{\prime}(z)\right| \leq \sup _{z \in \mathbb{D}} w(z)\left|\psi_{1}^{\prime}(z)-\psi_{2}^{\prime}(z)\right|\left|f\left(\phi_{1}(z)\right)\right| \\
+\sup _{z \in \mathbb{D}} w(z)\left|\psi_{2}^{\prime}(z)\right|\left|f\left(\phi_{1}(z)\right)-f\left(\phi_{2}(z)\right)\right| \\
+\sup _{z \in \mathbb{D}} w(z)\left|f^{\prime}\left(\phi_{1}(z)\right)\right|\left|\phi_{1}^{\prime}(z) \psi_{1}(z)-\phi_{2}^{\prime}(z) \psi_{2}(z)\right| \\
+\sup _{z \in \mathbb{D}} w(z)\left|\phi_{2}^{\prime}(z) \psi_{2}(z)\right|\left|f^{\prime}\left(\phi_{1}(z)\right)-f^{\prime}\left(\phi_{2}(z)\right)\right| \\
\leq \sup _{z \in \mathbb{D}} w(z)\left|\psi_{1}^{\prime}(z)-\psi_{2}^{\prime}(z)\right| \max \left\{\frac{1}{v\left(\phi_{1}(z)\right)}, \frac{1}{v\left(\phi_{2}(z)\right)}\right\}\|f\|_{v} \\
+\sup _{z \in \mathbb{D}} \max \left\{\left|\psi_{1}^{\prime}(z)\right|,\left|\psi_{2}^{\prime}(z)\right|\right\} \max \left\{\frac{1}{v\left(\phi_{1}(z)\right)}, \frac{1}{v\left(\phi_{2}(z)\right)}\right\} \rho\left(\phi_{1}(z), \phi_{2}(z)\right)\|f\|_{v} \\
+\sup _{z \in \mathbb{D}} \max \left\{\frac{w(z)}{u\left(\phi_{1}(z)\right)}\left\|f^{\prime}\right\|_{u}\left|\phi_{1}^{\prime}(z) \psi_{1}(z)-\phi_{2}^{\prime}(z) \psi_{2}(z)\right|\right. \\
\leq \sup _{z \in \mathbb{D}} w(z)\left|\psi_{1}^{\prime}(z)-\psi_{2}^{\prime}(z)\right| \max \left\{\frac{1}{u\left(\phi_{2}(z)\right)}\right\} \rho\left(\phi_{1}(z), \phi_{2}(z)\right)\left\|f^{\prime}\right\|_{u} \\
+\sup _{z \in \mathbb{D}} \max \left\{\left|\psi_{1}^{\prime}(z)\right|,\left|\psi_{2}^{\prime}(z)\right|\right\} \max \left\{\frac{1}{v\left(\phi_{1}(z)\right)}, \frac{1}{v\left(\phi_{2}(z)\right)}\right\} \rho\left(\phi_{1}(z), \phi_{2}(z)\right)\|f\|_{v} \\
+\sup _{z \in \mathbb{D}} \max \left\{\frac{w(z)}{u\left(\phi_{1}(z)\right)}, \frac{w(z)}{u\left(\phi_{2}(z)\right)}\right\}\|D\|\|f\|_{v}\left|\phi_{1}^{\prime}(z) \psi_{1}(z)-\phi_{2}^{\prime}(z) \psi_{2}(z)\right| \\
+\sup _{z \in \mathbb{D}} \max \left\{\frac{w(z)}{u\left(\phi_{1}(z)\right)}, \frac{w(z)}{u\left(\phi_{2}(z)\right)}\right\} \rho\left(\phi_{1}(z), \phi_{2}(z)\right)\|D\|\|f\|_{v}
\end{array}
$$

and the claim follows.

Next, we turn our attention to compactness of $\psi_{1} C_{\phi_{1}}-\psi_{2} C_{\phi_{2}}: H_{v}^{\infty} \rightarrow B_{w}$.

Proposition 6. Let $w$ be a weight and $v$ be a weight as described in the beginning of this section. Let $\psi_{1}, \psi_{2} \in H(\mathbb{D})$ and $\phi_{1}, \phi_{2}$ be analytic self-maps of $\mathbb{D}$. If $\psi_{1} C_{\phi_{1}}-\psi_{2} C_{\phi_{2}}: H_{v}^{\infty} \rightarrow B_{w}$ is bounded, then the following conditions are satisfied 
(a) $\lim \sup _{\left|\phi_{1}(z)\right| \rightarrow 1} w(z)\left|\frac{\psi_{1}^{\prime}(z)}{v\left(\phi_{1}(z)\right)^{\frac{1}{2}}} \varphi_{\phi_{2}(z)}^{2}\left(\phi_{1}(z)\right)+2 \frac{\psi_{1}(z)}{v\left(\phi_{1}(z)\right)^{\frac{1}{2}}} \varphi_{\phi_{2}(z)}\left(\phi_{1}(z)\right) \varphi_{\phi_{2}(z)}^{\prime}\left(\phi_{1}(z)\right)\right|=0$,

(b) $\lim \sup _{\left|\phi_{2}(z)\right| \rightarrow 1} w(z)\left|\frac{\psi_{2}^{\prime}(z)}{v\left(\phi_{2}(z)\right)^{\frac{1}{2}}} \varphi_{\phi_{1}(z)}^{2}\left(\phi_{2}(z)\right)+2 \frac{\psi_{2}(z)}{v\left(\phi_{2}(z)\right)^{\frac{1}{2}}} \varphi_{\phi_{1}(z)}\left(\phi_{2}(z)\right) \varphi_{\phi_{1}(z)}^{\prime}\left(\phi_{2}(z)\right)\right|=0$,

(c) $\lim \sup _{\left|\phi_{1}(z)\right| \rightarrow 1}\left|\frac{\psi_{1}(z) w(z) \overline{\phi_{1}(z)} \nu^{\prime}\left(\left|\phi_{1}(z)\right|^{2}\right)}{v\left(\phi_{1}(z)\right)}\right| \rho\left(\phi_{1}(z), \phi_{2}(z)\right)=0$,

(d) $\lim \sup _{\left|\phi_{2}(z)\right| \rightarrow 1}\left|\frac{\psi_{2}(z) w(z) \overline{\phi_{2}(z)} \nu^{\prime}\left(\left|\phi_{2}(z)\right|^{2}\right)}{v\left(\phi_{2}(z)\right)}\right| \rho\left(\phi_{1}(z), \phi_{2}(z)\right)=0$,

Proof (a) Consider a sequence $\left(z_{n}\right)_{n} \subset \mathbb{D}$ such that $\left|\phi_{1}\left(z_{n}\right)\right| \rightarrow 1$ if $n \rightarrow \infty$. We set

$$
f_{\phi_{1}\left(z_{n}\right)}(z):=v_{\phi_{1}\left(z_{n}\right)}\left(\phi_{1}\left(z_{n}\right)\right)^{\frac{1}{6}}\left(\frac{3}{2} \frac{1}{v_{\phi_{1}\left(z_{n}\right)}(z)^{2}}-\frac{v_{\phi_{1}\left(z_{n}\right)}\left(\phi_{1}\left(z_{n}\right)\right)}{v_{\phi_{1}\left(z_{n}\right)}(z)^{3}}\right)^{\frac{1}{3}}
$$

and

$$
g_{\phi_{1}\left(z_{n}\right)}(z):=f_{\phi_{1}\left(z_{n}\right)}(z) \varphi_{\phi_{2}\left(z_{n}\right)}^{2}(z) \text { for every } z \in \mathbb{D} \text {. }
$$

Thus $\left\|g_{\phi_{1}\left(z_{n}\right)}\right\|_{v} \leq \sup _{z \in \mathbb{D}} v_{\phi_{1}\left(z_{n}\right)}\left(\phi_{1}\left(z_{n}\right)\right)^{\frac{1}{6}}\left|\frac{3}{2} \frac{v(z)^{3}}{v_{\phi_{1}\left(z_{n}\right)}(z)^{2}}-\frac{v(z)^{3} v_{\phi_{1}\left(z_{n}\right)}\left(\phi_{1}\left(z_{n}\right)\right)}{v_{\phi_{1}\left(z_{n}\right)}(z)^{3}}\right|^{\frac{1}{3}} \leq M^{\frac{1}{6}}\left(\frac{5}{2} M\right)^{\frac{1}{3}}$ for every $n \in \mathbb{N}$, where $M:=\sup _{z \in \mathbb{D}} v(z)$. Thus, $\left(g_{\phi_{1}\left(z_{n}\right)}\right)_{n \in \mathbb{N}}$ is a bounded sequence in $H_{v}^{\infty}$ which converges to zero uniformly on the compact subsets of $\mathbb{D}$. Moreover,

$$
g_{\phi_{1}\left(z_{n}\right)}^{\prime}(z)=f_{\phi_{1}\left(z_{n}\right)}^{\prime}(z) \varphi_{\phi_{2}\left(z_{n}\right)}^{2}(z)+2 f_{\phi_{1}\left(z_{n}\right)}(z) \varphi_{\phi_{2}\left(z_{n}\right)}(z) \varphi_{\phi_{2}\left(z_{n}\right)}^{\prime}(z) \text { for every } z \in \mathbb{D}
$$

where

$$
\begin{array}{r}
f_{\phi_{1}\left(z_{n}\right)}^{\prime}(z)=v_{\phi_{1}\left(z_{n}\right)}\left(\phi_{1}\left(z_{n}\right)\right)^{\frac{1}{6}}\left(\frac{3}{2} \frac{1}{v_{\phi_{1}\left(z_{n}\right)}(z)^{2}}-\frac{v_{\phi_{1}\left(z_{n}\right)}\left(\phi_{1}\left(z_{n}\right)\right)}{v_{\phi_{1}\left(z_{n}\right)}(z)^{3}}\right)^{-\frac{2}{3}} . \\
\cdot\left(-\frac{v_{\phi_{1}\left(z_{n}\right)}^{\prime}(z)}{v_{\phi_{1}\left(z_{n}\right)}(z)^{3}}+\frac{v_{\phi_{1}\left(z_{n}\right)}\left(\phi_{1}\left(z_{n}\right)\right)}{v_{\phi_{1}\left(z_{n}\right)}(z)^{4}} v_{\phi_{1}\left(z_{n}\right)}^{\prime}(z)\right)
\end{array}
$$

for every $n \in \mathbb{N}$. By Proposition 3, the fact that $\psi_{1} C_{\phi_{1}}-\psi_{2} C_{\phi_{2}}$ is compact yields

$$
\left\|\left(\psi_{1} C_{\phi_{1}}-\psi_{2} C_{\phi_{2}}\right) g_{\phi_{1}\left(z_{n}\right)}\right\|_{B_{w}} \rightarrow 0 \text { if } n \rightarrow \infty
$$

Finally,

$$
\begin{aligned}
& \left\|\left(\psi_{1} C_{\phi_{1}}-\psi_{2} C_{\phi_{2}}\right) g_{\phi_{1}\left(z_{n}\right)}\right\|_{B_{w}} \geq \\
& w\left(z_{n}\right)\left|\frac{\psi_{1}^{\prime}\left(z_{n}\right)}{v\left(\phi_{1}\left(z_{n}\right)\right)^{\frac{1}{2}}} \varphi_{\phi_{2}\left(z_{n}\right)}^{2}\left(\phi_{1}\left(z_{n}\right)\right)+2 \frac{\psi_{1}\left(z_{n}\right) \varphi_{\phi_{2}\left(z_{n}\right)}\left(\phi_{1}\left(z_{n}\right)\right) \varphi_{\phi_{2}\left(z_{n}\right)}^{\prime}\left(\phi_{1}\left(z_{n}\right)\right)}{v\left(\phi_{1}\left(z_{n}\right)\right)^{\frac{1}{2}}}\right|
\end{aligned}
$$

Thus, (a) follows and we can show (b) analogously. Consider now

$$
f_{\phi_{1}\left(z_{n}\right)}(z):=\frac{v_{\phi_{1}\left(z_{n}\right)}\left(\phi_{1}\left(z_{n}\right)\right)}{v_{\phi_{1}\left(z_{n}\right)}(z)}-\left(\frac{v_{\phi_{1}\left(z_{n}\right)}\left(\phi_{1}\left(z_{n}\right)\right)}{v_{\phi_{1}\left(z_{n}\right)}(z)}\right)^{\frac{1}{2}}=\frac{v\left(\phi_{1}\left(z_{n}\right)\right)}{v_{\phi_{1}\left(z_{n}\right)}(z)}-\left(\frac{v\left(\phi_{1}\left(z_{n}\right)\right)}{v_{\phi_{1}\left(z_{n}\right)}(z)}\right)^{\frac{1}{2}}
$$

and

$$
g_{\phi_{1}\left(z_{n}\right)}(z):=f_{\phi_{1}\left(z_{n}\right)}(z) \varphi_{\phi_{2}\left(z_{n}\right)}^{2}(z) \text { for every } z \in \mathbb{D}
$$




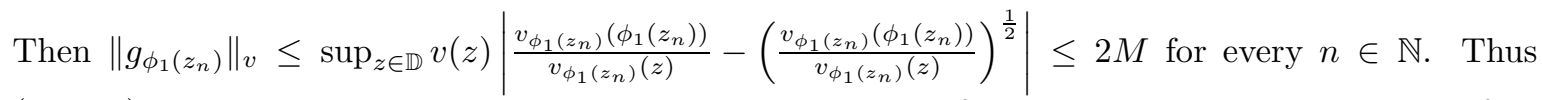
$\left(g_{\phi_{1}\left(z_{n}\right)}\right)_{n}$ is a bounded sequence in $H_{v}^{\infty}$ and $g_{\phi_{1}\left(z_{n}\right)} \rightarrow 0$ uniformly on every compact subset of $\mathbb{D}$. Moreover $g_{\phi_{1}\left(z_{n}\right)}\left(\phi_{1}\left(z_{n}\right)\right)=0$ and $g_{\phi_{1}\left(z_{n}\right)}^{\prime}\left(\phi_{1}\left(z_{n}\right)\right)=-\frac{1}{2} \frac{v_{\phi_{1}\left(z_{n}\right)}^{\prime}\left(\phi_{1}\left(z_{n}\right)\right)}{v\left(\phi_{1}\left(z_{n}\right)\right)} \varphi_{\phi_{2}\left(z_{n}\right)}^{2}\left(\phi_{1}\left(z_{n}\right)\right)$. Since $\psi_{1} C_{\phi_{1}}-$ $\psi_{2} C_{\phi_{2}}$ is compact, by Proposition 3 we have $\left\|\left(\psi_{1} C_{\phi_{1}}-\psi_{2} C_{\phi_{2}}\right) g_{n}\right\|_{B_{w}} \rightarrow 0$ if $n \rightarrow \infty$. Thus,

$$
\begin{aligned}
\left\|\left(\psi_{1} C_{\phi_{1}}-\psi_{2} C_{\phi_{2}}\right) g_{\phi_{1}\left(z_{n}\right)}\right\|_{B_{w}} & =\sup _{z \in \mathbb{D}} w(z)\left|\left(\left(\psi_{1} C_{\phi_{1}}-\psi_{2} C_{\phi_{2}}\right) g_{\phi_{1}\left(z_{n}\right)}\right)^{\prime}(z)\right| \\
& \geq \frac{1}{2} w\left(z_{n}\right)\left|\psi_{1}\left(z_{n}\right) \phi_{1}^{\prime}\left(z_{n}\right) \overline{\phi_{1}\left(z_{n}\right)}\right| \rho\left(\phi_{1}\left(z_{n}\right), \phi_{2}\left(z_{n}\right)\right)^{2} \frac{\left|\nu^{\prime}\left(\left|\phi_{1}\left(z_{n}\right)\right|^{2}\right)\right|}{v\left(\phi_{1}\left(z_{n}\right)\right)} .
\end{aligned}
$$

Finally, $\lim \sup _{\left|\phi_{1}(z)\right| \rightarrow 1} w(z)|\psi(z)|\left|\phi_{1}^{\prime}(z)\right| \mid \overline{\phi_{1}(z) \mid} \frac{\left|\nu^{\prime}\left(\left|\phi_{1}(z)\right|^{2}\right)\right|}{v\left(\phi_{1}(z)\right)}=0$, and (c) holds. (d) follows analogously.

Proposition 7. Let $v$ and $w$ be weights. If

(a) there is a weight $u$ such that the operator $D: H_{v}^{\infty} \rightarrow H_{u}^{\infty}, f \rightarrow f^{\prime}$ is bounded and additionally $\limsup _{\max \left\{\left|\phi_{1}(z)\right|,\left|\phi_{2}(z)\right|\right\} \rightarrow 1} \max \left\{\frac{w(z)}{u\left(\phi_{1}(z)\right)}, \frac{w(z)}{u\left(\phi_{2}(z)\right)}\right\} \rho\left(\phi_{1}(z), \phi_{2}(z)\right)=0$ as well as $\limsup _{\max \left\{\left|\phi_{1}(z)\right|,\left|\phi_{2}(z)\right|\right\} \rightarrow 1} \max \left\{\frac{w(z)}{u\left(\phi_{1}(z)\right)}, \frac{w(z)}{u\left(\phi_{2}(z)\right)}\right\}\left|\phi_{1}^{\prime}(z) \psi_{1}(z)-\phi_{2}^{\prime}(z) \psi_{2}(z)\right|=0$,

(b) $\lim \sup _{\max \left\{\left|\phi_{1}(z)\right|, \phi_{2}(z) \mid\right\} \rightarrow 1} \max \left\{\frac{1}{v\left(\phi_{1}(z)\right)}, \frac{1}{v\left(\phi_{2}(z)\right)}\right\} w(z)\left|\psi_{1}^{\prime}(z)-\psi_{2}^{\prime}(z)\right|=0$,

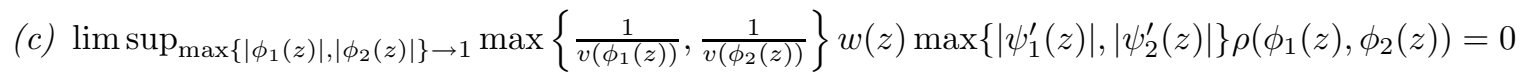
then $\psi_{1} C_{\phi_{1}}-\psi_{2} C_{\phi_{2}}: H_{v}^{\infty} \rightarrow B_{w}$ is compact.

Proof Let $\left(f_{n}\right)_{n \in \mathbb{N}}$ be a sequence in $H_{v}^{\infty}$ with $\left\|f_{n}\right\|_{v} \leq 1$ and $f_{n} \rightarrow 0$ uniformly on compact subsets of $\mathbb{D}$. By the assumption, for any $\varepsilon>0$ there is $0<\delta<1$ such that $\delta<\max \left\{\left|\phi_{1}(z)\right|,\left|\phi_{2}(z)\right|\right\}<1$ implies

$$
\begin{array}{r}
\max \left\{\frac{w(z)}{u\left(\phi_{1}(z)\right)}, \frac{w(z)}{u\left(\phi_{2}(z)\right)}\right\} \rho\left(\phi_{1}(z), \phi_{2}(z)\right)<\varepsilon \\
\max \left\{\frac{w(z)}{u\left(\phi_{1}(z)\right)}, \frac{w(z)}{u\left(\phi_{2}(z)\right)}\right\}\left|\phi_{1}^{\prime}(z) \psi_{1}(z)-\phi_{2}^{\prime}(z) \psi_{2}(z)\right|<\varepsilon \\
\max \left\{\frac{1}{v\left(\phi_{1}(z)\right)}, \frac{1}{v\left(\phi_{2}(z)\right)}\right\} w(z)\left|\psi_{1}^{\prime}(z)-\psi_{2}^{\prime}(z)\right|<\varepsilon \\
\max \left\{\frac{1}{v\left(\phi_{1}(z)\right)}, \frac{1}{v\left(\phi_{2}(z)\right)}\right\} w(z) \max \left\{\left|\psi_{1}^{\prime}(z)\right|,\left|\psi_{2}^{\prime}(z)\right|\right\} \rho\left(\phi_{1}(z), \phi_{2}(z)\right)<\varepsilon
\end{array}
$$


Then applying Lemma 1

$$
\begin{array}{r}
\sup _{z \in \mathbb{D}} w(z)\left|\left(\left(\psi_{1} C_{\phi_{1}}-\psi_{2} C_{\phi_{2}}\right) f_{n}\right)^{\prime}(z)\right| \leq \sup _{z \in \mathbb{D}} w(z)\left|\psi_{1}^{\prime}(z)-\psi_{2}^{\prime}(z)\right|\left|f_{n}\left(\phi_{1}(z)\right)\right| \\
+\sup _{z \in \mathbb{D}} w(z)\left|\psi_{2}^{\prime}(z)\right|\left|f_{n}\left(\phi_{1}(z)\right)-f_{n}\left(\phi_{2}(z)\right)\right| \\
+\sup _{z \in \mathbb{D}} w(z)\left|f_{n}^{\prime}\left(\phi_{1}(z)\right)\right|\left|\phi_{1}^{\prime}(z) \psi_{1}(z)-\phi_{2}^{\prime}(z) \psi_{2}(z)\right| \\
+\sup _{z \in \mathbb{D}} w(z)\left|\phi_{2}^{\prime}(z) \psi_{2}(z)\right|\left|f_{n}^{\prime}\left(\phi_{1}(z)\right)-f_{n}^{\prime}\left(\phi_{2}(z)\right)\right|
\end{array}
$$

The claim follows.

\section{Received: October 2008. Revised: February 2009.}

\section{References}

[1] K.D. Bierstedt, J. Bonet and J. Taskinen, Associated weights and spaces of holomorphic functions, Studia Math. 127 (1998), no. 2, 137-168.

[2] J. Bonet, P. Domański, M. Lindström and J. Taskinen, Composition operators between weighted Banach spaces of analytic functions, J. Austral. Math. Soc. Ser. A 64 (1998), no. 1, 101-118.

[3] J. Bonet, M. Lindström And E. Wolf, Differences of composition operators between weighted Banach spaces of holomorphic functions, J. Austral. Math. Soc. 84 (2008), no. 1, 9-20. 
[4] M.D. Contreras and A.G. Hernández-Díaz, Weighted composition operators in weighted Banach spaces of analytic functions, J. Austral. Math. Soc. Ser. A 69 (2000), no. 1, 41-60.

[5] C. Cowen and B. MacCluer, Composition Operators on Spaces of Analytic Functions, CRC Press, Boca Raton, 1995.

[6] P. Domański and M. Lindström, Sets of interpolation and sampling for weighted Banach spaces of holomorphic functions, Ann. Plon. Math. 79 (2002), no. 3, 233-264.

[7] A. Harutyunyan and W. Lusky, On the boundedness of the differentiation operator between weighted spaces of holomorphic functions, Studia Math. 184 (2008), no. 3, 233-247.

[8] T. Hosokawa, K. Izuchi and S. Ohno, Topological structure of the space of weighted compositon operators on $H^{\infty}$, Integral Equations Operator Theory 53 (2005), no. 4, 509-526.

[9] M. Lindström And E. Wolf, Essential norm of the difference of weighted composition operators, Monatshefte Math. 153 (2008), no. 2, 133-143.

[10] W. Lusky, On weighted spaces of harmonic and holomorphic functions, J. London Math. Soc. 51 (1995), no. 2, 309-320.

[11] W. Lusky, On the isomorphism classes of weighted spaces of harmonic and holomorphic functions, Studia Math. 175 (2006), no. 1, 19-45.

[12] B. MacCluer, S. Ohno And R. Zhao, Topological structure of the space of composition operators on $H^{\infty}$, Integral Equations Operator Theory 40 (2001), no. 4, 481-494.

[13] S. Ohno, Weighted composition operators between $H^{\infty}$ and the Bloch space, Taiwanese J. Math. 5 (2001), no. 3, 555-563.

[14] S. Ohno, K. Stroethoff and R. Zhao, Weighted composition operators between Bloch type spaces, Rocky Mountain J. Math. 33 (2003), no. 1, 191-215.

[15] J.H. ShapIro, Composition Operators and Classical Function Theory, Springer, 1993.

[16] E. Wolf, Differences of composition operators between weighted Banach spaces of holomorphic functions on the unit polydisk, Results Math. 51 (2008), no. 3-4, 361-372

[17] E. Wolf, Compact differences of composition operators, Bull. Aust. math. Soc. 77 (2008), no. 1, 161-165.

[18] E. Wolf, Differences of weighted composition operators, to appear in Collectanea Math. 